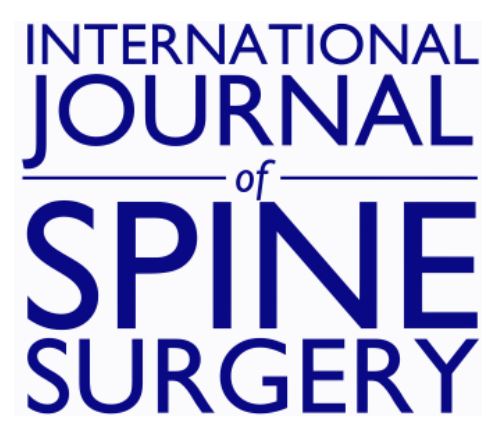

\title{
L5 - S1 Segmental Kinematics After Facet Arthroplasty
}

Leonard I. Voronov, Robert M. Havey, David M. Rosler, Simon G. Sjovold, Susan L. Rogers, Gerard Carandang, Jorge A. Ochoa, Hansen Yuan, Scott Webb and Avinash G. Patwardhan

Int J Spine Surg 2009, 3 (2) 50-58

doi: https://doi.org/10.1016/SASJ-2009-0001-RR

http://ijssurgery.com/content/3/2/50

This information is current as of April 26, 2023.

Email Alerts Receive free email-alerts when new articles cite this article. Sign up at: http://ijssurgery.com/alerts 


\title{
L5 - S1 Segmental Kinematics After Facet Arthroplasty
}

Leonard I. Voronov, MD, PhD, ${ }^{a, b}$ Robert M. Havey, BS, ${ }^{a, b}$ David M. Rosler, MS, ${ }^{c}$ Simon G. Sjovold, MASc, ${ }^{c}$ Susan L. Rogers, MS, ${ }^{c}$ Gerard Carandang, BS, ${ }^{a}$ Jorge A. Ochoa, PhD, ${ }^{c}$ Hansen Yuan, MD, ${ }^{d}$ Scott Webb, DO, ${ }^{e}$ and Avinash G. Patwardhan, PhD ${ }^{a, b}$

\begin{abstract}
Background

Facet arthroplasty is a motion restoring procedure. It is normally suggested as an alternative to rigid fixation after destabilizing decompression procedures in the posterior lumbar spine. While previous studies have reported successful results in reproducing normal spine kinematics after facet replacement at L4-5 and L3-4, there are no data on the viability of facet replacement at the lumbosacral joint. The anatomy of posterior elements and the resulting kinematics at L5-S1 are distinctly different from those at superior levels, making the task of facet replacement at the lumbosacral level challenging. This study evaluated the kinematics of facet replacement at L5-S1.
\end{abstract}

\section{Methods}

Six human cadaveric lumbar spines (L1-S1, $46.7 \pm 13.0$ years) were tested in the following sequence: (1) intact (L1-S1), (2) complete laminectomy and bilateral facetectomy at L5-S1, and (3) implantation of TFAS-LS (Lumbosacral Total Facet Arthroplasty System, Archus Orthopedics, Redmond, Washington) at L5-S1 using pedicle screws. Specimens were tested in flexion $(8 \mathrm{Nm})$, extension $(6 \mathrm{Nm})$, lateral bending $(\mathrm{LB}, \pm 6 \mathrm{Nm})$, and axial rotation $(\mathrm{AR}, \pm 5 \mathrm{Nm})$. The level of significance was $a=.017$ after Bonferroni correction for three comparisons: (1) intact vs. destabilized, (2) destabilized vs. reconstructed, and (3) intact vs. reconstructed.

Results

Laminectomy-facetectomy at L5-S1 increased the L5-S1 angular range of motion (ROM) in all directions. Flexion-extension (F-E) ROM increased from $15.3 \pm 2.9$ to $18.7 \pm 3.5$ degrees $(P<.017)$, LB from $8.2 \pm 1.8$ to $9.3 \pm 1.6$ degrees $(P<.017)$, and AR from $3.7 \pm 2.0$ to $5.9 \pm 1.8$ degrees $(P<.017)$. The facet arthroplasty system decreased ROM compared to the laminectomy-facetectomy condition in all tested directions $(P<.017)$. The facet arthroplasty system restored the L5-S1 ROM to its intact levels in LB and AR $(P>.017)$. F-E ROM after the facet arthroplasty system implantation was smaller than the intact value $(10.1 \pm 2.2$ vs. $15.3 \pm 2.9$ degrees, $P<.017)$. The load-displacement curves after the facet arthroplasty system implantation at L5-S1 were sigmoidal, and quality of motion measures were similar to intact, demonstrating graded resistance to angular motion in F-E, LB and AR.

\section{Conclusions}

The facet arthroplasty system was able to restore stability to the lumbosacral segment after complete laminectomy and bilateral facetectomy, while also allowing near-normal kinematics in all planes. While F-E ROM after the facet arthroplasty system implantation was smaller than the intact value, it was within the physiologic norms for L5-S1. These results are consistent with previous studies of facet arthroplasty at L3-L4 and L4-L5 and demonstrate that TFAS technology can be adapted to the lumbosacral joint with functionality comparable to its application in superior lumbar levels.

Key Words: Spine, Biomechanics, Facet Arthroplasty. SAS Journal. June 2009;3:50-58. DOI: SASJ-2009-0001-RR

${ }^{a}$ Edward Hines Jr. VA Hospital, Hines, IL, 'boyola University Medical Center, Maywood, IL. cArchus Orthopedics, Inc., Redmond, WA 98052 dSUNY Syracuse, Syracuse, NY 13202, ${ }^{e}$ Florida Spine Institute, Pinellas Park, FL 33782

Investigation performed at the Musculoskeletal Biomechanics Laboratory, Edward Hines Jr. VA Hospital, Hines, Illinois

Address Correspondence to Avinash G. Patwardhan, PhD, Department of Orthopaedic Surgery and Rehabilitation, Loyola University Medical Center, 2160 S. First Avenue, Maywood, IL 60153 (e-mail: apatwar@lumc.edu)

Institutional research support provided by the Department of Veterans Affairs, Washington, D.C., and Archus Orthopedics, Inc., Redmond, Washington.

\section{INTRODUCTION}

Facet joints have been recognized as pain generators for nearly a century, ${ }^{1}$ but only recently have they garnered attention as joints that warrant functional replacement. Removal of some or all of the facets is often required during decompressive surgeries ${ }^{2,3}$ and in the treatment of degenerated facets or intervertebral discs. ${ }^{4}$ Until recently, the standard surgical intervention following compromise of the facet joint has been posterior fusion, which is usually augmented with pedicle screws. While 
this is a successful procedure with excellent clinical outcomes, there are a number of potential long-term drawbacks. Adjacent segment degeneration often occurs above the fused segment, which may require additional surgery. In addition, the immobilizing effects of fusion do not restore normal function and mechanics of the decompressed segment, the ramifications of which are not well understood. The risk of accelerated degeneration adjacent to these fused levels has increased interest in the preservation of motion via arthroplasty. Facet replacement has been proposed as an alternative to fusion and instrumentation after laminectomy for spinal stenosis.

Current clinical trials are investigating the safety and effectiveness of facet replacement technologies, and the kinematic function of these devices has been reported in the literature..$^{5-8}$ However, these devices are indicated for treatment only at L3-4 and L4-5 levels. There is clinical need for facet replacement technology for the L5-S1 level due to the potentially high incidence of iatrogenic injuries $^{2}$ and degenerative changes ${ }^{1,9}$ of the L5-S1 facet joints. However, the geometry ${ }^{2,10,11}$ and $\operatorname{loads}^{1}$ on the L5-S1 facet joints are different from those on the more superior levels and, as such, a level-specific implant is required for the L5-S1 level.

A facet replacement implant for the lumbosacral joint (TFAS-LS [Lumbo-Sacral Total Facet Arthroplasty System], Archus Orthopedics, Redmond, Washington) has been designed to address the facet morphology and loading at this level. It allows for replacement of the resected facets when either maintaining the lamina or following partial or total laminectomy and facetectomy. Each of two caudal socket-type bearings is connected to the sacrum via two sacral screws. Cephalad arms, with spherical bearings located at the proper position on the caudal bearings, are connected to L5 pedicle screws. A crossarm is used to securely link the two cephalad arms (Figures 1 and 2). The profile of the articulating surface of the the facet arthroplasty system caudal bearings is specifically designed so that it guides the motion at the implanted level by providing graduated resistance to motion of the spherical bearings attached to the cephalad vertebral body. Relative ramp angles are incorporated into the caudal bearings so that in flexion-extension, lateral bending, and axial rotation the resistance to angular motion increases as the motion increases.

The goal of the study was to assess the kinematics of L5S1 segments implanted with the facet replacement device to determine its ability to restore the function of the facet joints and associated resected posterior structures.

\section{MATERIALS AND METHODS}

Specimens and Experimental Set-up

Six fresh-frozen human cadaveric spines from L1 to sacrum (age: $46.7 \pm 13.0$ years; 3 males, 3 females) with

\section{Figure 1.}

\section{SASJournal}

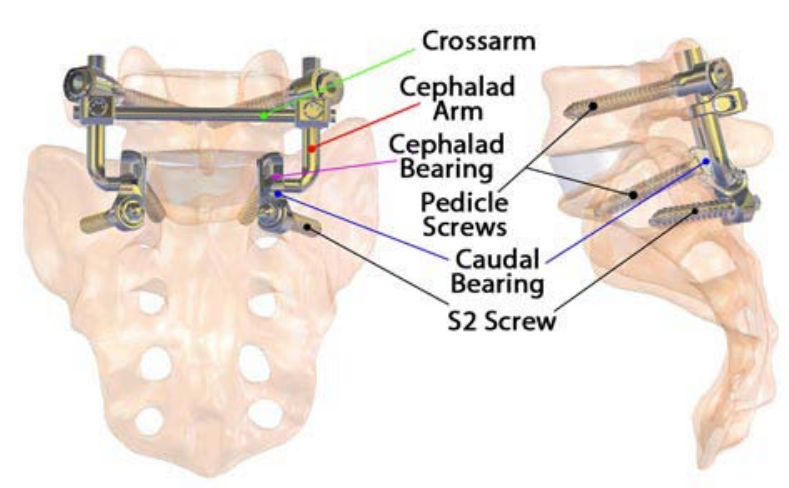

Lumbosacral Total Facet Arthroplasty System (TFAS-LS). A computeraided illustration in a functional spinal unit of a facet prosthesis with labeling of individual components: posterior view (left) and lateral view (right).

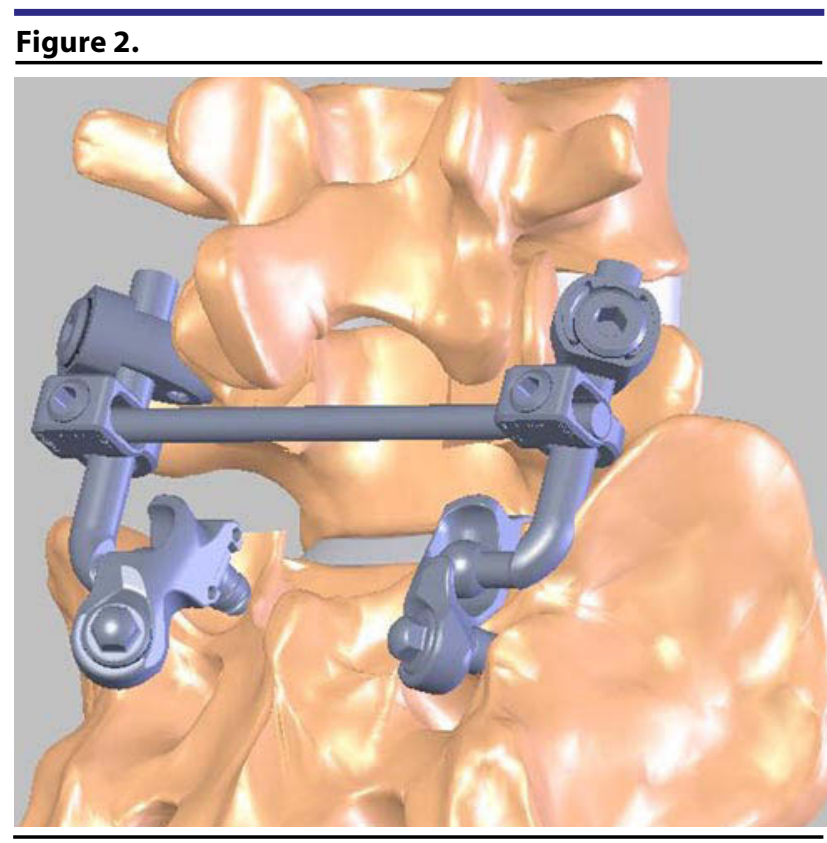

Isometric view of computer-aided illustration of TFAS-LS in a functional spinal unit.

no previous spinal surgery were used. Specimens were screened radiographically to exclude those with evidence of disc ossification and bridging osteophytes. The specimens had minimal to mild facet hypertrophy, and none of the specimens was osteopenic or osteoporotic. The specimens were thawed at room temperature $(20$ $\left.{ }^{\circ} \mathrm{C}\right) 24$ hours before testing. The paravertebral muscles were dissected, while leaving the discs, ligaments and posterior bony structures intact. All tests were performed at room temperature and the specimens were kept moist during testing with saline soaked towels. The L1 vertebra and sacrum were anchored in cups using bone cement and pins.

Each specimen was mounted on a 6-component load cell (model MC3A-6-250, AMTI multiaxis transducers, 


\section{SASJournal}

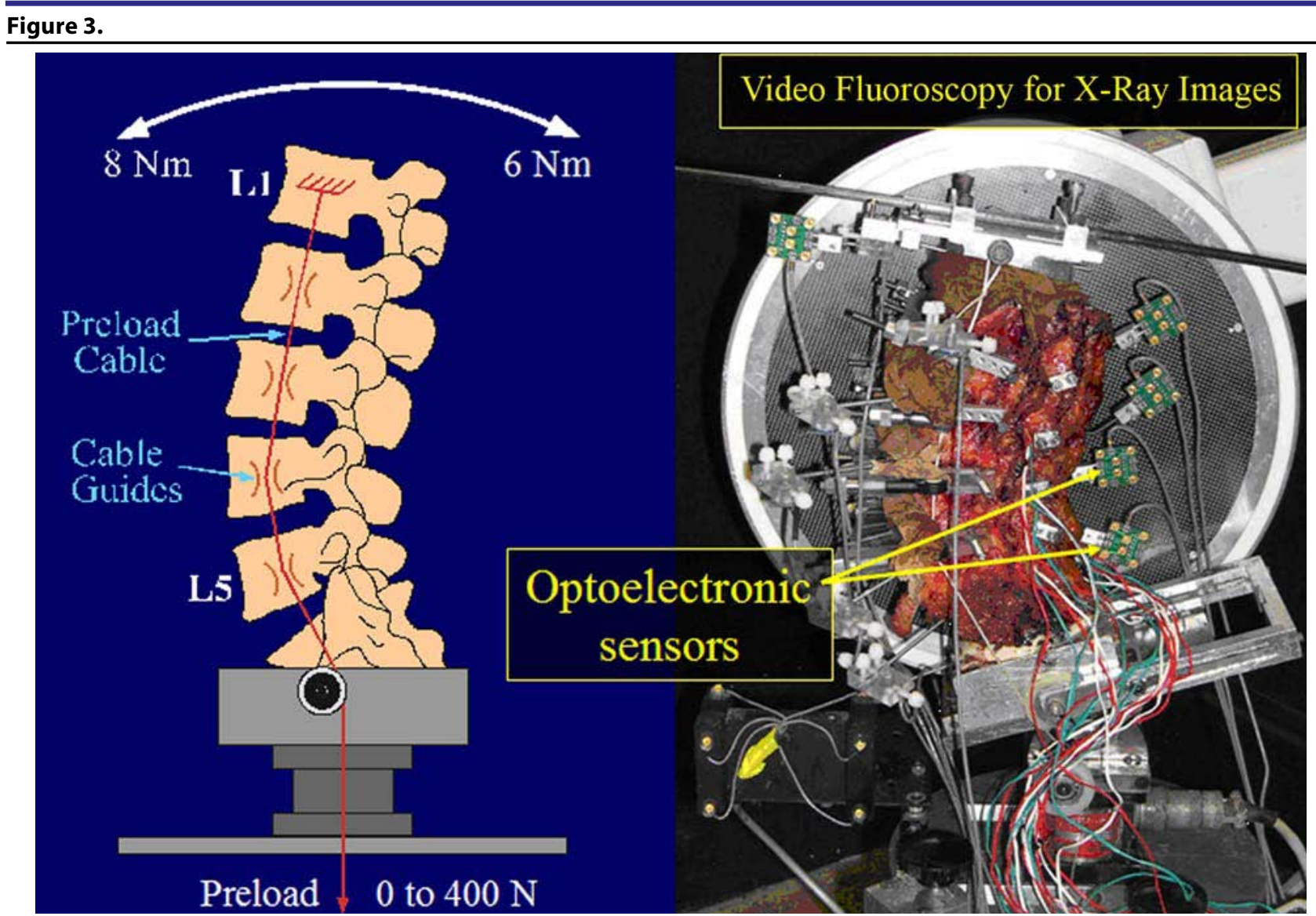

Experimental set-up:schematic (left) and specimen photo (right).

AMTI Inc., Watertown, Massachusetts) at the caudal end and was free to move in any plane at the proximal end. A moment was applied by controlling the flow of water into bags attached to loading arms fixed to the L1 vertebra. The apparatus allowed continuous cycling of the specimen between specified maximum moment endpoints in flexion and extension, lateral bending, and axial rotation.

The motion of L1, L2, L3, L4, and L5 vertebrae relative to sacrum was measured using an optoelectronic motion measurement system (Optotrak model 3020, Northern Digital, Waterloo, Ontario, Canada). In addition, biaxial angle sensors (Applied Geomechanics, Santa Cruz, California) were mounted on each vertebra to provide real-time feedback for the optimization of the follower load path. Fluoroscopic imaging (GE OEC 9800 Plus digital fluoroscopy machine) was used during flexion and extension in order to monitor vertebra and implant motion.

The concept of follower load was used to apply compressive preload to the lumbar spine during flexion and extension. ${ }^{12}$ The compressive preload was applied along a path that followed the lordotic curve of the lumbar spine. By applying a compressive load along the follower load path, the segmental bending moments and shear forces due to the preload application were minimized. ${ }^{12}$ This allowed the lumbar spine to support physiologic compressive preloads without damage or instability. The preload was applied using bilateral loading cables that were attached to the cup holding the L1 vertebra (Figure 3 ). The cables passed freely through guides anchored to each vertebra and were connected to a loading hanger under the specimen. The cable guide mounts allowed anterior-posterior adjustments of the follower load path within a range of about $10 \mathrm{~mm}$. The preload path was optimized by adjusting the cable guides to minimize changes in lumbar lordosis when the compressive load (up to $400 \mathrm{~N}$ ) was applied to the specimen. Follower load was not applied during lateral bending and axial rotation, as with the current configuration it would have resulted in moments opposing the desired motion, giving erroneous results.

\section{Experimental Protocol}

Each specimen was subjected to flexion-extension, lateral bending and axial rotation in random order. The loaddisplacement behavior of the specimen was recorded under flexion moments up to $8 \mathrm{Nm}$ and extension moments up to $6 \mathrm{Nm} .{ }^{6,12}$ Lateral bending moments were recorded at $\pm 6 \mathrm{Nm}$, and axial rotation moments 


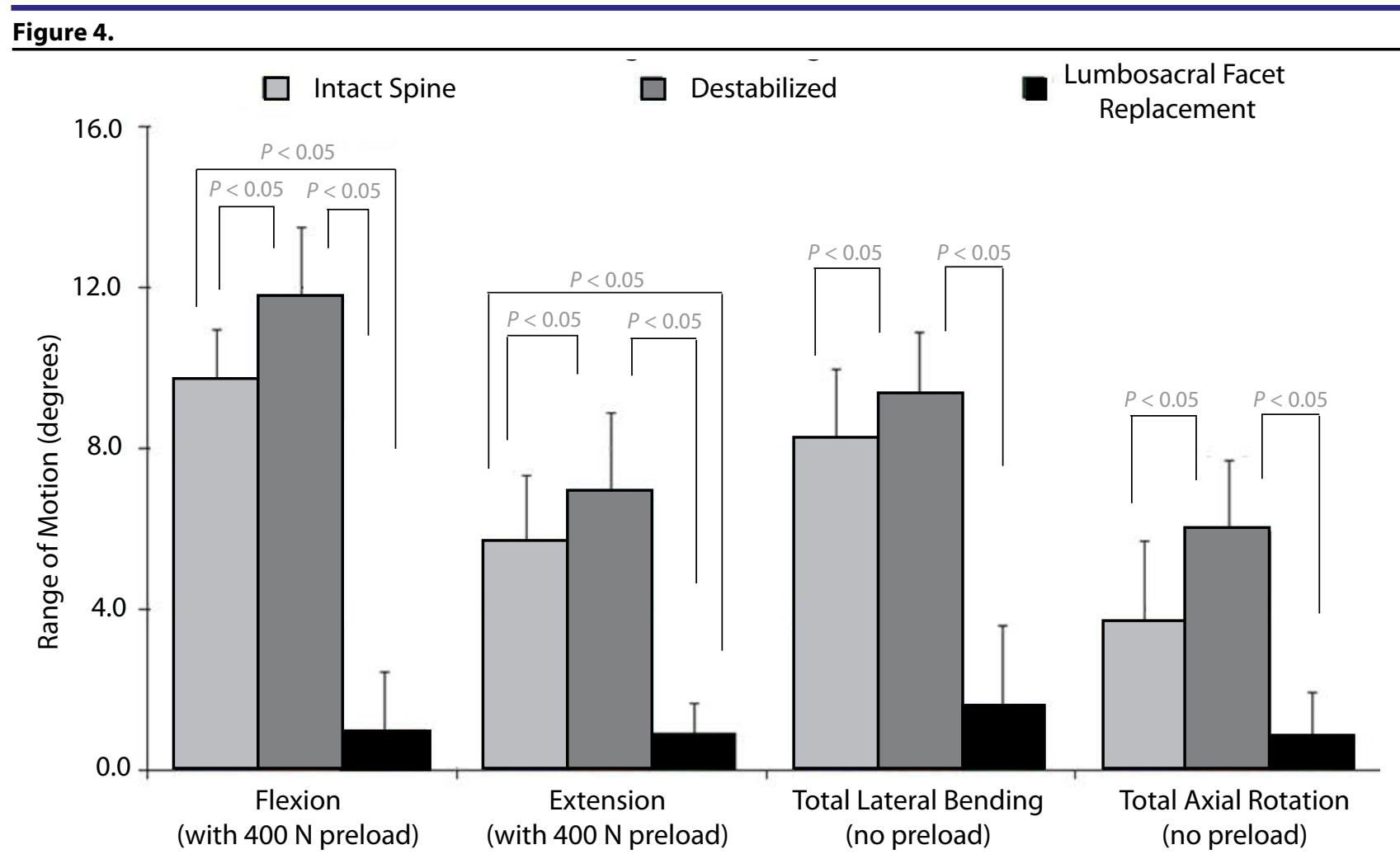

L5-S1 Segmental Range of Motion Data. Testing was conducted on segments that were intact, after complete L5 laminectomy and facetectomy, and after TFAS-LS implantation.

were recorded at $\pm 5 \mathrm{Nm}$. Flexion-extension was tested under $400 \mathrm{~N}$ preload. The load-displacement data were collected until two reproducible load-displacement loops were obtained. This required a maximum of three loading cycles.

Each specimen was tested in the following order: (1) intact, (2) after a complete L5 laminectomy and bilateral facetectomy at L5-S1, and (3) after implantation of the facet replacement prosthesis at L5-S1 using pedicle screws and secondary (S2) screws in the sacral construct. Fluoroscopy was used during the procedure to ensure proper sizing and placement of the device.

\section{Statistical Methods}

The load-displacement curves were analyzed to determine the L5-S1 angular range of motion (ROM) in flexion-extension, lateral bending, and axial rotation. Additionally, the L5-S1 segmental stiffness values (Nm/ degree) were calculated using previously described techniques. ${ }^{6}$ The statistical analysis was performed using repeated-measures analysis of variance (ANOVA, Systat Software Inc., Richmond, California). Post hoc tests were done using Bonferroni correction for multiple comparisons. The following pair-wise comparisons were made: (1) intact vs. destabilized, (2) destabilized vs. reconstructed, and (3) intact vs. reconstructed. The level of significance was $a=.017$ (after Bonferroni correction for 3 comparisons). This Bonferroni analysis was done separately for flexion-extension, lateral bending, and axial rotation data sets because no comparisons across the 3 load types were intended.

\section{RESULTS}

\section{Quantity of Motion}

Laminectomy and bilateral facetectomy at L5-S1 significantly increased the L5-S1 angular range of motion (ROM) in all directions. Flexion-extension ROM increased from $15.3 \pm 2.9$ to $18.7 \pm 3.5$ degrees $(P<.017)$, lateral bending ROM increased from $8.2 \pm 1.8$ to $9.3 \pm 1.6$ degrees $(P<.017)$, and axial rotation ROM increased from $3.7 \pm 2.0$ to $5.9 \pm 1.8$ degrees $(P<.017)$ (Figure 4$)$. The facet replacement device significantly decreased ROM compared to the laminectomyfacetectomy condition in all tested directions $(P<.017)$. Facet replacement restored the L5-S1 ROM to its intact levels in lateral bending and axial rotation $(P>.017)$. Total flexion-extension ROM after facet replacement implantation was smaller than the intact value (10.1 \pm 2.2 vs. $15.3 \pm 2.9$ degrees, $P<.017$ ), but it remained in physiologically normal ranges. Flexion and extension independently demonstrated the same statistical trend $(P$ $<.017$ ) as the total flexion-extension ROM.

\section{Quality of Motion}

The L5-S1 flexion stiffness in the high flexibility zone was significantly decreased after destabilization as compared to the intact segment $(0.37 \pm 0.11$ vs. 0.22 
$\pm 0.08 \mathrm{Nm} /$ degree; $P<.017)$. The facet replacement device significantly increased the stiffness from the surgically destabilized condition $(0.79 \pm 0.40$ vs. 0.22 $\pm 0.08 \mathrm{Nm} /$ degree; $P<.017)$, restoring it to the intact level $(P>.017)$. Similar results were found for motion response in lateral bending. The L5-S1 lateral bending stiffness in the high flexibility zone was significantly decreased after destabilization as compared to the intact value $(0.68 \pm 0.26$ vs. $0.47 \pm 0.22 \mathrm{Nm} /$ degree; $P<.017)$. Facet replacement significantly increased the stiffness from the surgically destabilized condition $(0.62 \pm 0.24$ vs. $0.47 \pm 0.22 \mathrm{Nm} / \mathrm{deg} ; P<.017)$, restoring it to the intact level $(P>.017)$. Additionally, the kinematic signatures after facet replacement implantation in the flexion-extension and lateral bending tests had similar sigmoid characteristics as the intact segment (Figure 5). The quality of motion was not assessed for axial rotation motion because of the relatively small values of total ROM in this mode.

\section{Figure 5.}

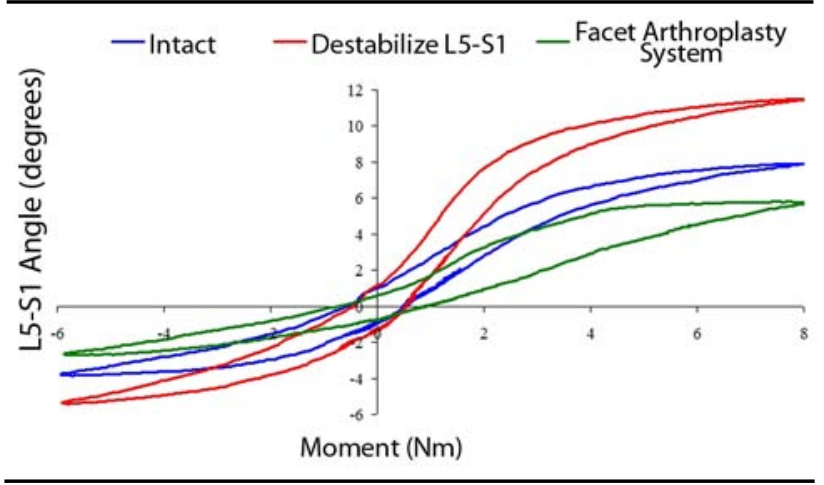

Load-Displacement Curves in Flexion-Extension Under $400 \mathrm{~N}$ Preload. Testing was conducted on segments that were intact, after complete L5 laminectomy and facetectomy, and after FAS implantation.

\section{DISCUSSION}

The lumbosacral level has the second highest incidence of degenerative disease in the lumbar spine, which suggests that there is a potentially large need for facet replacement as a treatment for "facetogenic" pain, destabilization associated with decompression, or in conjunction with an anterior motion preservation device. The sagittal tilt of the lumbosacral intervertebral disc space predisposes it to larger shear loads than the more superior levels. Additionally, this level is critical in controlling and limiting axial rotation. As such, a facet replacement for the lumbosacral joint must be capable of supporting large shear loads and properly limiting angular motions. This is the first study to evaluate the performance of a facet arthroplasty implant at the lumbosacral joint (facet replacement) in human cadaveric lumbar spines under physiologic loads. While this biomechanical study does not purport to address the clinical indications for the use of facet replacement, as facet replacement continues to be investigated in both the laboratory and the clinic, a better understanding of its benefits should become more evident and may allow for an expansion of the indications for its clinical use.

Due to the technical limitation of the current experimental set-up, a physiologic compressive preload was applied only while assessing the kinematics in flexionextension, not in lateral bending or axial rotation. The preload due to muscle activity has a stabilizing effect on a motion segment; therefore, the results pertaining to lateral bending and axial rotation may be viewed as a worst-case scenario. Theoretically, lower ROM values than those reported here for lateral bending and axial rotation may be anticipated in vivo under a physiologic preload.

Facet arthroplasty at L5-S1 using the facet replacement device maintained quantity and quality of motion at the operative level within physiologic values after wide decompressive laminectomy and bilateral facetectomy. While the flexion-extension motion was smaller with facet replacement than it was for the intact segment, it was within the physiologic norms for this level. As noted earlier, the shape and orientation of the facets at the L5-S1 level are different from those at the more superior levels. Their flatter shape and orientation closer to the dorsal plane demonstrate their critical function in preventing excessive axial rotation and shear. The facet replacement functioned similarly to the natural facets in this respect: Axial rotation and lateral bending motions were restored to the levels of intact following implantation of facet replacement at the destabilized level. Previous studies have demonstrated that facet replacement using TFAS technology restored both quantity and quality of motion after complete laminectomy and facetectomy at L4-5 $5^{5}$ and L3-4 ${ }^{6}$. In the current study, we performed the same surgical intervention and assessed the facet arthroplasty performance at L5-S1. The outcome of this study is similar to the previous studies: Physiologic range of motion and quality of motion were maintained in all tested directions. The graduated resistance to angular motion provided by the design of the facet replacement caudal bearings allows it to functionally replace the excised bone and soft tissues removed during wide decompressive laminectomy and facetectomy procedures. Similar to the natural elements, the resistance to motion (ie, stiffness of the functional spinal unit) increases with motion outside of the neutral zone.

In conclusion, the facet replacement implant was able to restore stability to the lumbosacral segment after complete laminectomy and bilateral facetectomy, while allowing near-normal motions in all planes. The TFAS technology originally designed for use in upper lumbar 
levels can be adapted to the lumbosacral joint without compromising spinal kinematics.

Facet replacement devices will require a substantial amount of validation testing and numerous clinical studies before they can be considered a viable treatment option for the treatment of spinal disorders. To date most pathophysiological research, and thus surgical treatments, has been focused on the disc as a pain generator. A more comprehensive focus on reestablishing the structure and function of the human functional spinal unit may include facet replacement. A better understanding of facet function and facet-mediated pain, possibly through classification of facet degeneration, may be needed in order to support the use of such devices.

This manuscript was submitted January 6, 2009, and was accepted April 15, 2009..

\section{REFERENCES}

1. Serhan HA, Varnavas G, Dooris AP, Patwardhan A, Tzsermiadonis M. Biomechanics of the posterior lumbar articulating elements. Neurosurg Focus. 2007;22(1):E1.

2. Celik SE, Celik S, Kara A, Goksu K. Lumbar facet joint angle and its importance on joint violation in lumbar microdiscectomy. Neurosurgery. 2008;62:168-173.

3. van Ooij A, Oner FC, Verbout AJ. Complication of artificial disc replacement: a report of 27 patients with the SB Charite Disc. $J$ Spinal Disord Tech. 2003;16(4):369-383.

4. Khoueir P, Kim A, Wang MY. Classification of posterior dynamic stabilization devices. Neurosurg Focus. 2007;22(1):E3.

5. Zhu Q, Larson CR, Sjovold SG, et al. Biomechanical evaluation of the Total Facet Arthroplasty System ${ }^{\mathrm{TM}}$ : 3-dimensional kinematics. Spine. 2007;32:1, 55-62.

6. Phillips FM, Tzermiadianos MN, Voronov LI, et al. Effect of the Total Facet Arthroplasty System after complete laminectomy-facetectomy on the biomechanics of implanted and adjacent segments. Spine $J$. Jan-Feb 2009;9(1):96-102.

7. Goel VK, Mehta A, Jangra J, et al. Anatomic Facet Replacement System (AFRS) restoration of lumbar segment mechanics to intact: a finite element study and in vitro cadaver investigation. SAS Journal. 2007;1(1):46-54.

8. Wilke H-J, Schmidt H, Werner K, Schmolz W, Drumm J. Biomechanical evaluation of a new total posterior-element replacement system. Spine. 2006;31(24):2790-2796.

9. Cohen SP, Raja SN. Pathogenesis, diagnosis, and treatment of lumbar zygapophysial (facet) joint pain. Anesthesiology. 2007;106:591614.

10. Rousseau M-A, Bradford DS, Hadi TM, Pederson KL, Lotz JC. The instant axis of rotation influences facet forces at $\mathrm{L} 5 / \mathrm{S} 1$ during flexion/ extension and lateral bending. Eur Spine J. 2006;15:299-307.

11. Don AS, Robertson PA. Facet joint orientation in spondylolysis and isthmic spondylolisthesis. J Spinal Disord Tech. 2008;21(2):112115 .
12. Patwardhan AG, Havey RM, Meade KP, Lee B, Dunlap B. A follower load increases the load-carrying capacity of the lumbar spine in compression. Spine. 1999;24:1003-1009. 


\section{EXTENDED REFERENCES}

Effect of the Total Facet Arthroplasty System after complete laminectomy-facetectomy on the biomechanics of implanted and adjacent segments.

Phillips FM, Tzermiadianos MN, Voronov LI, Havey $R M$, Carandang $G$, Renner SM, Rosler DM, Ochoa JA, Patwardhan $A G$.

BACKGROUND CONTEXT: Lumbar fusion is traditionally used to restore stability after wide surgical decompression for spinal stenosis. The Total Facet Arthroplasty System (TFAS) is a motionrestoring implant suggested as an alternative to rigid fixation after complete facetectomy. PURPOSE: To investigate the effect of TFAS on the kinematics of the implanted and adjacent lumbar segments. STUDY DESIGN: Biomechanical in vitro study. METHODS: Nine human lumbar spines (L1 to sacrum) were tested in flexion-extension $(+8$ to $-6 \mathrm{Nm})$, lateral bending $(+/-$ $6 \mathrm{Nm})$, and axial rotation $(+/-5 \mathrm{Nm})$. Flexion-extension was tested under $400 \mathrm{~N}$ follower preload. Specimens were tested intact, after complete L3 laminectomy with L3-L4 facetectomy, after L3-L4 pedicle screw fixation, and after L3-L4 TFAS implantation. Range of motion (ROM) was assessed in all tested directions. Neutral zone and stiffness in flexion and extension were calculated to assess quality of motion. RESULTS: Complete laminectomy-facetectomy increased L3-L4 ROM compared with intact in flexion-extension $(8.7+/-$ 2.0 degrees to $12.2+/-3.2$ degrees, $\mathrm{p}<.05)$ lateral bending $(9.0+/-2.5$ degrees to $12.6+/-3.2$ degrees, $\mathrm{p}=$ $.09)$, and axial rotation $(3.8+/-2.7$ degrees to $7.8+/-4.5$ degrees $\mathrm{p}<.05)$. Pedicle screw fixation decreased ROM compared with intact, resulting in $1.7+/-0.5$ degrees flexion-extension $(\mathrm{p}<.05), 3.3+/-1.4$ degrees lateral bending $(\mathrm{p}<.05)$, and $1.8+/-0.6$ degrees axial rotation $(\mathrm{p}=.09)$. TFAS restored intact ROM $(\mathrm{p}>.05)$ resulting in 7.9+/-2.1 degrees flexion-extension, 10.1+/-3.0 degrees lateral bending, and 4.7+/-1.6 degrees axial rotation. Fusion significantly increased the normalized ROM at all remaining lumbar segments, whereas TFAS implantation resulted in near-normal distribution of normalized ROM at the implanted and remaining lumbar segments. Flexion and extension stiffness in the high-flexibility zone decreased after facetectomy $(\mathrm{p}<.05)$ and increased after simulated fusion $(\mathrm{p}<.05)$. TFAS restored quality of motion parameters (loaddisplacement curves) to intact $(\mathrm{p}>.05)$. The quality of motion parameters for the whole lumbar spine mimicked L3-L4 segmental results. CONCLUSIONS: TFAS restored range and quality of motion at the operated segment to intact values and restored nearnormal motion at the adjacent segments.

Biomechanical evaluation of the Total Facet Arthroplasty System: 3-dimensional kinematics.

Zhu $Q$, Larson CR, Sjovold $S G$, Rosler DM, Keynan $O$, Wilson DR, Cripton PA, Oxland TR.
STUDY DESIGN: An in vitro biomechanical study to quantify 3-dimensional kinematics of the lumbar spine following facet arthroplasty. OBJECTIVES: To compare the multidirectional flexibility properties and helical axis of motion of the Total Facet Arthroplasty System (TFAS) (Archus Orthopedics, Redmond, WA) to the intact condition and to posterior pedicle screw fixation. SUMMARY OF BACKGROUND DATA: Facet arthroplasty in the lumbar spine is a new concept in the field of spinal surgery. The kinematic behavior of any complete facet arthroplasty device in the lumbar spine has not been reported previously. METHODS: Flexibility tests were conducted on 13 cadaveric specimens in an intact and injury model, and after stabilization with the TFAS and posterior pedicle screw fixation at the L4-L5 level. A pure moment of $+/-10 \mathrm{Nm}$ with a compressive follower preload of $600 \mathrm{~N}$ was applied to the specimen in flexionextension, axial rotation, and lateral bending. Range of motion (ROM), neutral zone, and helical axis of motion were calculated for the L4-L5 segment. RESULTS: ROM with the TFAS was $81 \%$ of intact in flexion $(\mathrm{P}=0.035)$, $68 \%$ in extension $(\mathrm{P}=0.079), 88 \%$ in lateral bending $(\mathrm{P}=$ $0.042)$, and $128 \%$ in axial rotation $(P=0.013)$. The only significant change in neutral zone with TFAS compared to the intact was an increase in axial rotation $(\mathrm{P}=0.011)$. The only significant difference in helical axis of motion location or orientation between the TFAS and intact condition was an anterior shift of the helical axis of motion in axial rotation $(\mathrm{P}=0.013)$. CONCLUSIONS: The TFAS allowed considerable motion in all directions tested, with ROM being less than the intact in flexion and lateral bending, and greater than the intact in axial rotation. The helical axis of motion with the TFAS was not different from intact in flexion-extension and lateral bending, but it was shifted anteriorly in axial rotation. The kinematics of the TFAS were more similar to the intact spine than were the kinematics of the posterior fixation when applied to a destabilized lumbar spine.

Anatomic Facet Replacement System (AFRS) restoration of lumbar segment mechanics to intact: a finite element study and in vitro cadaver investigation

Vijay K. Goel, PhD, Ankit Mehta, BS, Jayant Jangra, BS, Ahmed Faizan, BS, Ali Kiapour, MS, Robert W. Hoy, MEng, and Andrew R. Fauth, PhD

BACKGROUND: Many decompression procedures involve complete or partial facetectomy. Spinal fusion usually stabilizes the motion segment after complete facetectomies. However, problems with fusion, such as adjacent-level degeneration, have increased interest in motion preservation technologies. Facet arthroplasty may become an important posterior motion-preservation device, but its biomechanical literature is sparse. METHODS: We conducted an in vitro investigation and finite element study to compare the biomechanical effects of an artificial facet system to the intact spine. In the in vitro study, we tested human osteo-ligamentous segments (L3-S1) in intact, injured, and artificial facet-repaired 
conditions. For the finite element study, we used a 3dimensional ligamentous L3-S1 segment model. We simulated destabilization in the intact model by removing the facets across the L4-L5 functional unit, then repaired it with appropriately sized facet implants and compared the ranges of motion, facet loads, disc pressures, and device loads. We also analyzed a finite element model with a rigid posterior pedicle-rod fixation system. We subjected the cadaveric specimens and the models to 400 $\mathrm{N}$ of follower load plus a $10 \mathrm{Nm}$ moment in extension, flexion, bending, and rotation. We used a novel technique to apply the follower load in the finite element models such that preload induced minimal vertebral rotation during the range of motion. RESULTS: The predicted ranges of motion for the intact and implanted models were consistent with cadaver data. After destabilization and facet replacement, the artificial facet system restored motion in all loading modes to intact values. The implant facet loads were similar to intact facet loads in extension and axial rotation, but less in lateral bending. The intradiscal pressure at the implanted level for the facet replacement device was similar to the intact pressure, whereas with the rigid system the intradiscal pressure was up to $70 \%$ less than the intact pressure. The maximum von-Mises stress predicted in the facet replacement construct was $85 \mathrm{MPa}$ in extension at the bone-pedicle screw interface, compared with $174 \mathrm{MPa}$ in the rigid system. Contact stresses at implant mating surfaces were minimal. CONCLUSIONS: The artificial facet system replicated natural facet kinematics. The cadaveric ranges of motion and the predicted fi nite element-based data indicated that the implant can "restore" the normal function of the segment after artificial facet replacement. CLINICAL RELEVANCE: Compared to rigid posterior pedicle-rod fixation, the artificial facet system restored the intact mechanics at the implanted level and may prevent adjacent-level degeneration.

Biomechanical evaluation of a new total posteriorelement replacement system.

\section{Wilke HJ, Schmidt H, Werner K, Schmölz W, Drumm J.}

STUDY DESIGN: In vitro study to characterize the flexibility of a new total posterior-element system when instrumented to L4-L5 segments. OBJECTIVE: The goal of this in vitro study was to investigate whether an optimized version of the TOPS implant (Impliant Ltd., Ramat Poleg, Israel) is capable to restore the physiologic motion characteristic of a spinal segment after facetectomy. SUMMARY OF BACKGROUND DATA: The TOPS implant is designed to replace the posterior elements of a functional spinal unit, to provide flexible restabilization and spinal alignment, while maintaining the intervertebral disc. The implant is composed of bilateral pedicle screws, connected with 2 crossbars in the transversal plane. The crossbars are joined together by an elastic element capable of transmitting tensile and compressive loads, as well as shear forces. METHODS: Six human cadaver specimens (L3-S1) (median age 61 years: minimum 47 and maximum 74 years) were used for this in vitro experiment. The specimens were loaded with pure moments of $+/-7.5 \mathrm{Nm}$ in flexion/extension, lateral bending, and axial rotation. The following states were investigated: (1) intact; (2) after bilateral laminectomy, including facetectomy of the lower facet joints, of the upper vertebra L4; and (3) after device implantation. The range of motion (ROM), neutral zone, and intradiscal pressure were determined from a third cycle. In a second step, the ROM in axial rotation was determined as a function of different flexion/ extension postures. RESULTS: In the neutral position, the laminectomy and facetectomy increased the median values of the ROM in flexion plus extension, lateral bending right plus left, and significantly in axial rotation left plus right from: 8.2 degrees, 7.6 degrees, 3.6 degrees to 12.1 degrees, 8.5 degrees, and 8.5 degrees (Wilcoxon signed rank test; $\mathrm{P}<0.05)$. After fixation of the implant, the ROM was again reduced to 6.8 degrees, 7.8 degrees, and 3.8 degrees. In a flexed posture, the ROM in axial rotation was slightly increased compared to the neutral position. With increasing extension, the axial rotation decreased linearly from 3.7 degrees in neutral position to 2.3 degrees in 4 degrees extension in the segment L4L5. The characteristic of the intradiscal pressure versus load with the implant was similar to that of the intact specimen. CONCLUSION: The TOPS implant almost ideally restored the ROM in lateral bending and axial rotation compared to that of the intact specimen. In the sagittal plane, $85 \%$ of the intact ROM could be obtained. The ROM in axial rotation as a function of flexion and extension angle also mimics the biomechanical behavior of the posterior complex of a lumbar spine. This relationship between ROM and posture emphasizes the importance of a proper implantation.

\section{A follower load increases the load-carrying capacity of the lumbar spine in compression.}

Patwardhan AG, Havey RM, Meade KP, Lee B, Dunlap B.

STUDY DESIGN: An experimental approach was used to test human cadaveric spine specimens. OBJECTIVE: To assess the response of the whole lumbar spine to a compressive follower load whose path approximates the tangent to the curve of the lumbar spine. SUMMARY OF BACKGROUND DATA: Compression on the lumbar spine is $1000 \mathrm{~N}$ for standing and walking and is higher during lifting. Ex vivo experiments show it buckles at 80-100 N. Differences between maximum ex vivo and in vivo loads have not been satisfactorily explained. METHODS: A new experimental technique was developed for applying a compressive follower load of physiologic magnitudes up to $1200 \mathrm{~N}$. The experimental technique applied loads that minimized the internal shear forces and bending moments, made the resultant internal force compressive, and caused the load path to approximate the tangent to the curve of the lumbar spine. RESULTS: A compressive vertical load applied in the neutral lordotic and forward-flexed postures caused large changes in lumbar lordosis at 


\section{SASJournal}

small load magnitudes. The specimen approached its extension or flexion limits at a vertical load of $100 \mathrm{~N}$. In sharp contrast, the lumbar spine supported a load of up to $1200 \mathrm{~N}$ without damage or instability when the load path was tangent to the spinal curve. CONCLUSIONS: Until this study, an experimental technique for applying compressive loads of in vivo magnitudes to the whole lumbar spine was unavailable. The load-carrying capacity of the lumbar spine sharply increased under a compressive follower load, as long as the load path remained within a small range around the centers of rotation of the lumbar segments. The follower load path provides an explanation of how the whole lumbar spine can be lordotic and yet resist large compressive loads. This study may have implications for determining the role of trunk muscles in stabilizing the lumbar spine. 